Article

\title{
Amplitude Modulation And Nonlinear Self-Interactions of the Geodesic Acoustic Mode at the Edge of MAST
}

\author{
Bogdan Hnat ${ }^{1, *(\mathbb{D}}$, Nicholas Walkden ${ }^{2} \mathbb{D}$ and The MAST Team ${ }^{2}$ \\ 1 Physics Department, University of Warwick, Coventry CV4 7AL, UK \\ 2 EURATOM/CCFE Fusion Association, Culham Science Centre, Abingdon OX14 3DB, UK; \\ Nick.Walkden@ukaea.uk \\ * Correspondence: b.hnat@warwick.ac.uk
}

Received: 1 April 2019; Accepted: 1 May 2019; Published: 8 May 2019

check for updates

\begin{abstract}
We studied the amplitude modulation of the radial electric field constructed from the Langmuir probe plasma potential measurements at the edge of the mega-ampere spherical tokamak (MAST). The Empirical Mode Decomposition (EMD) technique was applied, which allowed us to extract fluctuations on temporal scales of plasma turbulence, the Geodesic Acoustic Mode (GAM), and those associated with the residual poloidal flows. This decomposition preserved the nonlinear character of the signal. Hilbert transform (HT) was then used to obtain the amplitude modulation envelope of fluctuations associated with turbulence and with the GAM. We found significant spectral coherence at frequencies between 1-5 $\mathrm{kHz}$, in the turbulence and the GAM envelopes and for the signal representing the low frequency zonal flows (LFZFs). We present the evidence of local and nonlocal, in frequency space, three wave interactions leading to coupling between the GAM and the low frequency (LF) part of the spectrum.
\end{abstract}

Keywords: fusion plasma; radial electric field; temporal intermittency

\section{Introduction}

The edge region of tokamaks, defined by the steep pressure gradient, is dominated by turbulent structures of density, temperature, and the electrostatic potential (see, for example, Reference [1] and references therein) arising from resistive and/or interchange plasma instabilities. These fluctuations are responsible for the turbulent radial transport, which drives anomalous heat and particle losses and has a detrimental impact on the global energy confinement. Thus, better understanding and control of the edge transport is fundamental to enabling enhanced plasma confinement scenarios of future fusion reactors. The anisotropic shear amplification of micro-turbulence Reynolds stress produces radially localised, toroidally and poloidaly symmetric flows, called zonal flows (ZFs). These flows are distinct from the residual poloidal flows, often called zero frequency ZFs [2,3]. Shearing associated with both types of ZFs can nonlinearly modify stability threshold of unstable plasma modes [4-6] and reduces turbulence level by vortex stretching [7-9].

ZFs are axisymmetric electrostatic potential modes with zero poloidal and toroidal numbers, $m=n=0$. In tokamak geometry, toroidal curvature couples the ZF to the density perturbations with poloidal mode numbers $m \geq 1(n=0)$, and with a finite frequency. This compressible component of ZF is called the Geodesic Acoustic Mode (GAM). The local dispersion relation for the GAM has been derived from various plasma models and the leading term is $\omega_{G} \sim c_{S} / R_{0}$, where $c_{S}$ is the local sound speed and $R_{0}$ 
is the major radius $[10,11]$. The amplitude of the density fluctuations varies with the poloidal angle $\theta$ as $A \sim \sin (\theta)$. Since its theoretical prediction, the GAM has been experimentally observed in many tokamaks [12-16]. While the local dispersion relation suggests a monotonic change of the GAM frequency with radius, due to temperature gradient, there is growing evidence that the GAM is a global mode with a complex radial mode structure [17-20]. Driven by turbulent fluctuations [21], ZF and GAM provide a natural sink for turbulent energy. The stability of these flows is less understood. The GAM is damped by a Landau mechanism, in the region of low safety factor $q(r)$. The ZF/GAM can decay via nonlinear tertiary Kelvin-Helmholtz (K-H) instability of small scale fluctuations [5,21]. Nonlinear advection of GAM pressure perturbations provides a mechanism for the energy transport from ZF back to micro-turbulence scales [22,23].

Nonlinear interaction of ZF/GAM with turbulence are fundamental to our understanding of the L-H transition [8,24]. In this context, GAM is a valuable tool in the experimental studies of ZF due to its finite frequency, which allows easier identification of ZF in experimental data [19]. Here, we studied a possible mechanism for the generation of the LF GAM envelope by nonlinear self-interaction and the coupling of GAM/ZF and turbulence via these LF amplitude modulations. We analyzed the oscillating radial electric field component on different temporal scales. The power distribution of the radial electric field oscillations at GAM frequency was not uniform in time. Instead, it showed strong temporal intermittency, that is, the power was concentrated in few intense temporal regions, separated by intervals of low level activity. We used Hilbert transform (HT) based techniques to extract radial electric field fluctuations on different temporal scales, while preserving their nonlinear character. This allowed us to construct signals representing meso-scale turbulence, the oscillatory GAM signal, and the LFZFs, with frequencies in the range of few $\mathrm{kHz}$. HT gave nonlinear envelopes for the turbulence and the GAM. The spectral coherence of the turbulence with the GAM was then examined. We found that the amplitude modulation of the turbulence and the GAM had a similar behavior at LFs, between $2-5 \mathrm{kHz}$. The auto bi-coherence revealed nonlinear self-interaction of GAM and the possible coupling to these LF components.

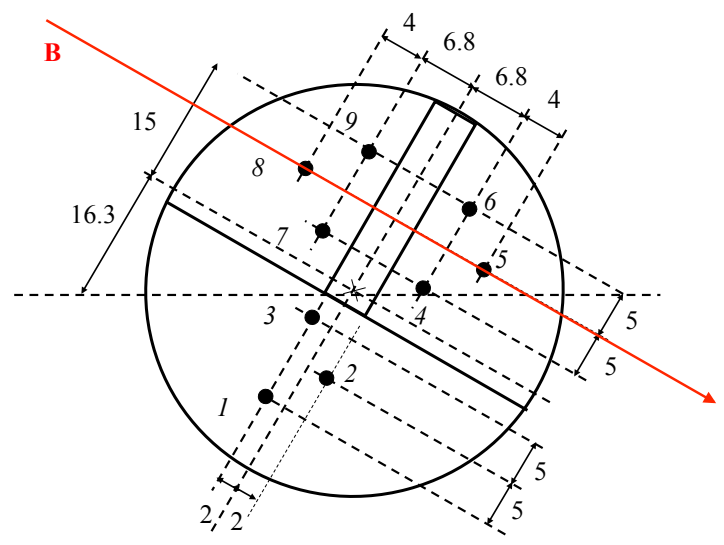

Figure 1. Langmuir Mach probe with separations of pins in millimeters. Pins 1, 2, and 3 are radially offset by $8 \mathrm{~mm}$ from all other pins. Red arrow indicates the average direction of the total magnetic field vector at the edge of mega-ampere spherical tokamak (MAST) for a typical L mode discharge.

\section{Experimental Setup and Data}

The MAST had a major radius $R_{0} \approx 0.85 \mathrm{~m}$, a minor radius $a \approx 0.65 \mathrm{~m}$, and the magnetic field strength $B=0.5 \mathrm{~T}$. The magnetic field vector pitch angle in the L-mode was about $22^{\circ}$ at the edge of the 
device. We analyzed data from an Ohmic L-mode plasma discharge numbered 29150 with no additional heating power and with a double null magnetic configuration. A line average number density of the plasma was $n \approx 1.47 \times 10^{19} \mathrm{~m}^{-3}$, and plasma current $I_{p}=0.43 \mathrm{MA}$.

A Mach type reciprocating Langmuir probe [25], positioned on the outboard mid-plane, measured floating potential, $\tilde{V}_{f}$, as well as a set of ion saturation currents (pins 2, 5, and 8). The schematic of the probe is shown in Figure 1. Pin pair $(1,3)$ was positioned $8 \mathrm{~mm}$ behind pairs $(4,6)$ and $(7,9)$. The floating potential $\tilde{V}_{f}$ was assumed to be a good approximation of the plasma potential $\tilde{V}_{p}$, and these were related by $\tilde{V}_{p}=\tilde{V}_{f}+\Lambda$. Here, $\Lambda$ was a sheath potential drop, which is usually approximated by $\Lambda \approx 2.5 T_{e} / e$ [26]. Thus, we assumed that the electrostatic potential fluctuations were larger than those in electron/ion temperature. The measurement of $\tilde{V}_{f}$ should have been weakly effected by the sheath potential of the Mach barrier, since it was based on the ion and electron currents balance, which was a function of the plasma temperature only. The high values of temporal correlations on all pin pairs were consistent with these assumptions.

We constructed radial electric field component $E_{r}$ by differencing the floating potential values on pin pair $(1,9), E_{r}=\nabla_{r} \tilde{V}_{p} \approx\left(\tilde{V}_{p}^{1}-\tilde{V}_{p}^{9}\right) / d_{r}^{(1,9)}$, where the superscripts on the floating potential indicated a pin and $d_{r}^{(1,9)}=8 \mathrm{~mm}$ is a radial separation of these pins. We noted that the poloidal separation of the pin pair $(1,9), d_{\theta}^{(1,9)}=3.8 \mathrm{~cm}$, was much larger than the radial separation. The radial electric field $E_{r}$ estimations from other radially separated pin pairs exhibited similar spectral features, but had smaller amplitude oscillations at the frequency expected for the GAM, in comparison with turbulent fluctuations. We chose a time interval of $0.315-0.33 \mathrm{~s}$, during which the intermittent character of the most powerful fluctuations is clearly present.

Figure 2 presents a summary of the data. Panel (a) shows the time series of the radial electric field $E_{r}(t)$, containing 7500 samples. Assuming that the toroidal magnetic field is dominant, the radial electric field gives the poloidal flow speed $v_{\theta}=\left(E_{r} B_{\phi}\right) / B^{2}$. Panel $(\mathrm{b})$ shows the distance of the Mach probe in relation to the last closed flux surface (LCFS) during this time interval. The probe is inside the plasma its radial depth varies approximately between -2 and $-4 \mathrm{~cm}$. Panel (c) of Figure 2 shows electron temperature from Thomson scattering diagnostic [27] at $0.333 \mathrm{~s}$, which is the closest measurement to the interval studied here. Each separate profile was taken at time $t=0.333+k \cdot 0.25 \times 10^{-5} \mathrm{~s}, k \in[0,7]$. These give the median of electron temperature at the probe location of $T_{e} \approx 14 \mathrm{eV}$. The GAM frequency depends on the electron temperature through the local sound speed value, $c_{s} \approx \sqrt{T_{e} / m_{p}}$, where $m_{p}$ is the proton mass. The GAM frequency obtained from the fluid-based dispersion relation for the MAST edge plasma parameters [15] was been calculated to be $f_{G} \approx 10 \mathrm{kHz}$ at the electron temperature $T_{e}=30 \mathrm{eV}$ and $f_{G} \approx 6$ $\mathrm{kHz}$ at $T_{e}=10 \mathrm{eV}$. The proton gyroradius at $T_{e}=10 \mathrm{eV}$ is $\rho_{p}=0.15 \mathrm{~cm}$. 

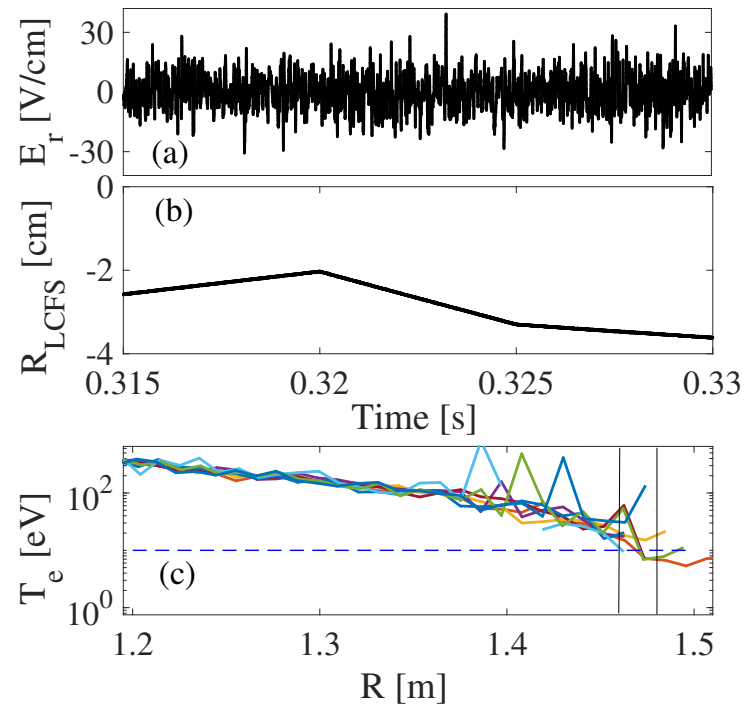

Figure 2. Summary of the data. (a) Wavelet power at lower frequencies. (b) Mach probe distance from the last closed flux surface (LCFS); negative values indicate position inside the plasma. (c) Eight electron temperature profiles from Thompson scattering system, at times $t=0.333+k \cdot 0.25 \times 10^{-5} \mathrm{~s}$, with $k \in[0,7]$. Two vertical lines indicate an approximate range of Mach probe positions. The dash horizontal line indicates the temperature of $10 \mathrm{eV}$.

\section{Methods}

The methods based on the HT offer a natural approach to studying temporal intermittency in a time series [28]. The intermittency is interpreted as an amplitude modulation of a mode, or a group of modes, of interest. Here, we employed this method as an effective filter, which allowed us to construct signals representing different dynamical temporal scales. The HT performs well when analyzing non-stationary and non-harmonic fluctuations arising in nonlinear systems. Fourier-based spectral techniques, as well as the wavelet transform, are unsuitable for such a time series if the principal aim is to preserve the nonlinear nature of the wave trains. The HT makes use of the Empirical Mode Decomposition (EMD) [29], which expands the input signal onto a set of intrinsic mode functions (IMFs) derived directly from the data.

The EMD is implemented as the iterative process. Cubic splines are used to connect the local maxima and local minima of the signal, forming the upper and the lower envelopes of the data. The mean of these envelopes, $m_{1}$, is calculated. For an input signal $S(t)$, the difference, $h_{1}=S(t)-m_{1}$ gives the first estimation of the envelope of $S(t)$. In the case of a nonlinear signal, this envelope's mean is, in general, not equal to the true local mean. The process is therefore repeated $k$ times until the resultant, $h_{1 k}$, satisfies the requirement for an IMF, $h_{1(k-1)}-m_{1 k}=h_{1 k}$, where $h_{1 k}$ and $m_{1 k}$ are the first envelope and its mean after $k$ th iteration, accordingly. We then designate $s_{1}(t)=h_{1 k}$ as the first IMF component of the data, containing the shortest period of the signal. Fluctuations at this scale are removed from the data to obtain a residual $r_{1}=S(t)-s_{1}(t)$. The procedure is then repeated for the residual $r_{1}$, treated as a new input signal. The decomposition is stopped either when the component $s_{i}$, or the residue $r_{i}$, becomes too small to be of interest, or when the residue, $r_{i}$, becomes a monotonic function from which no more IMFs can be 
extracted. For data with a trend, the final residue should be that trend. When the process is finished, we obtain the decomposition of a signal $S(t)$ into IMFs $s_{i}$ and the final residue:

$$
S(t)=\sum_{i=1}^{N} s_{i}(t)+r_{N}
$$

An individual IMF may contain oscillations with different periods, and different IMFs can contain similar periods. This spectral leakage, or mode mixing, can be an issue, especially for short and intermittent data. We incorporate the ensemble empirical mode decomposition (EEMD) $[30,31]$ to reduce the impact of mode mixing. This noise-assisted method adds white noise to the original data before the iterative process starts. The EMD modes are computed as normal until all of the IMFs are calculated. The original data is then reprocessed with a different noise realization, and the final IMF is averaged over all ensembles.

In this work, we used EEMD to decompose the radial electric field time series into a number of IMFs. We were interested in the amplitude modulation of turbulence and the GAM. The modes with periods shorter than that of the GAM are interpreted as turbulence. An envelope of a modulated signal can be constructed using analytic signal $S_{a}$ :

$$
S_{a}(t)=S(t)+i \mathcal{H}[S(t)]=E(t) \exp [i \phi(t)]
$$

where $\mathcal{H}[S(t)]$ indicates the HT of the signal $S$, and $i^{2}=-1$. For a slowly modulated signal, the modulus of $S_{a}$ corresponds to the amplitude modulation envelope $E(t)$. The frequency of $S_{a}$, which may not be be constant in time, can be obtained from the mean of the instantaneous phase change $f=\langle d \phi / d t\rangle$.

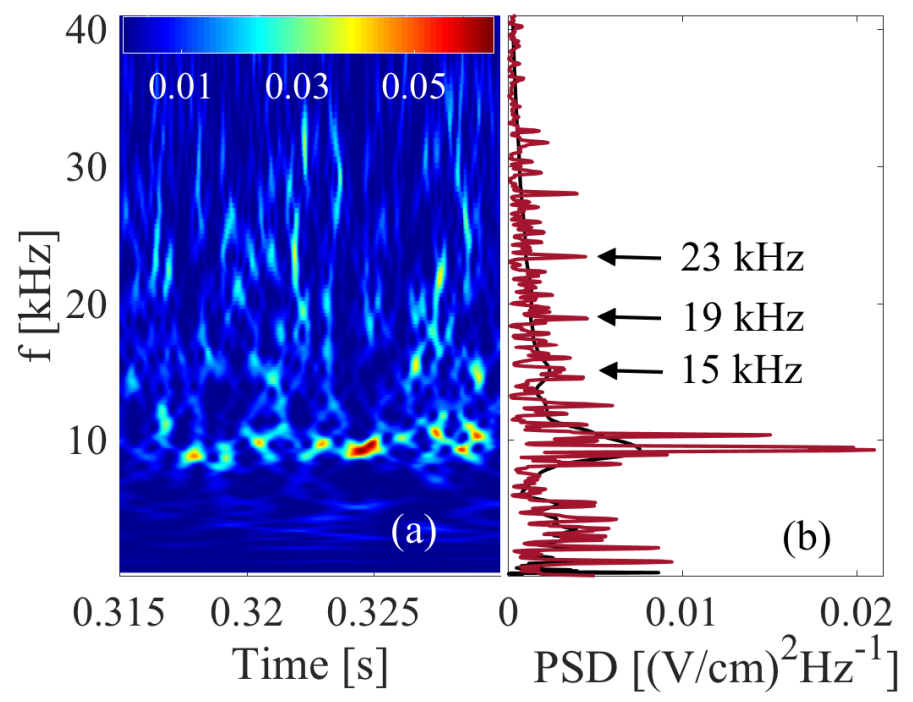

Figure 3. (a) Wavelet dynamic spectrum of $E_{r}(t)$. (b) Integrated wavelet power spectrum (black) and Fourier spectrum estimate (red). A significant spectral peak at $\sim 10 \mathrm{kHz}$ is clearly seen. Fourier spectrum shows an internal structure of the GAM peak, with multiple modes separated by $\sim 1 \mathrm{kHz}$. 
In order to quantify the nonlinear interactions between different modes, we used the wavelet bi-coherence defined as:

$$
b^{2}\left(f_{1}, f_{2}\right)=\frac{\left|\left\langle\tilde{S}\left(f_{1}, \tau\right) \tilde{S}\left(f_{2}, \tau\right) \tilde{S}^{*}\left(f_{1}+f_{2}, \tau\right)\right\rangle\right|^{2}}{\left\langle\left|\tilde{S}\left(f_{1}, \tau\right) \tilde{S}^{*}\left(f_{2}, \tau\right)\right|^{2}\right\rangle\left\langle\left|\tilde{S}\left(f_{1}+f_{2}, \tau\right)\right|^{2}\right\rangle},
$$

where $\tilde{S}$ is a wavelet coefficient at a scale associated with a period $1 / f$ and at time $\tau$. For a signal $S(t)$, the wavelet coefficients are given by:

$$
\tilde{S}(s, \tau)=\int_{-\infty}^{\infty} d t S(t) \frac{1}{\sqrt{s}} \psi^{*}\left(\frac{t-\tau}{s}\right),
$$

where $s$ is a temporal scale, $\tau$ is a new time label and $\psi(t)$ is the analyzing wavelet. We use Bump wavelets [32], which have better frequency resolution, but poorer time localisation compared with a standard Morlet wavelet. Given a set of wavelet coefficients $\tilde{S}(f, \tau)$ the wavelet spectrum estimate is given by [33]:

$$
P(f)=\left\langle\tilde{S}^{*}(\nu, \tau) \tilde{S}(\nu, \tau)\right\rangle_{\tau} .
$$

Wavelet estimates of the bi-coherence are superior to these obtained form the Fourier transform for shorter and non-stationary data sets. Fourier-based bi-coherence requires the averaging over many realizations of the same data, while the averaging indicated by $\langle\ldots\rangle$ in Equation (3) is over time.

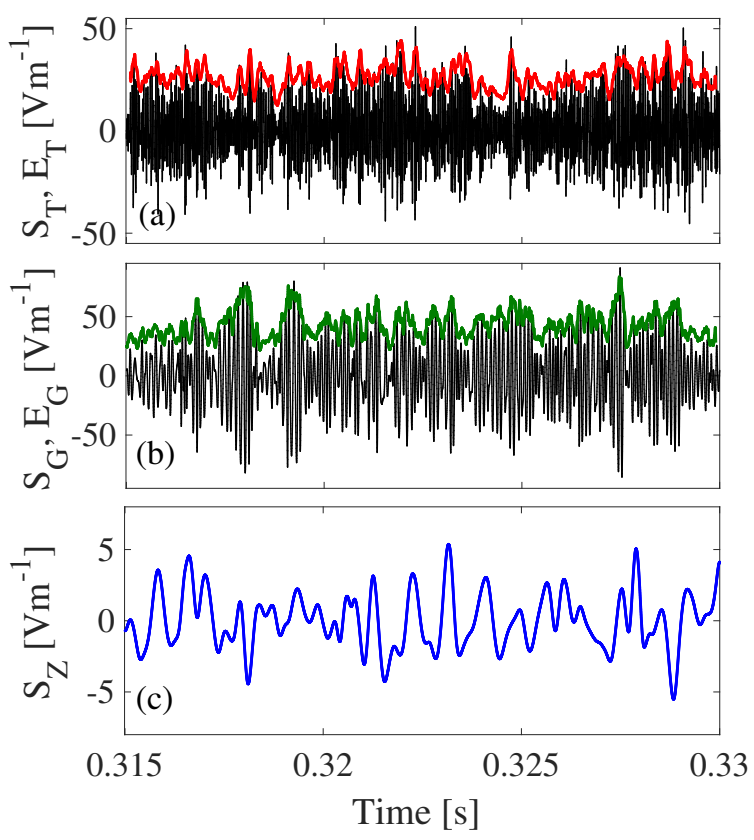

Figure 4. Signals constructed from ensemble empirical mode decomposition (EEMD) produced intrinsic mode functions (IMFs): (a) turbulence $S_{T}$ (black) and its envelope $E_{T}$ smoothed over consecutive 50 points (red), (b) the GAM signal $S_{G}$ (black) and its envelope $E_{G}$ smoothed over consecutive 30 points (green), and (c) low frequency zonal flows (LFZFs) $S_{Z}$ (blue). Envelopes of turbulence $E_{T}$ and of the GAM were offset vertically for clarity by 10 units. 


\section{Results}

A strong oscillatory component has been previously identified in MAST edge plasma density and electrostatic potential fluctuations measured by the reciprocating Langmuir probes $[15,20]$. This mode showed a spectral power peak at the frequency of $\sim 10 \mathrm{kHz}$, in reasonably good agreement with the theoretical and numerical predictions for GAM frequency in MAST L-mode edge plasma $[15,16]$. Figure 3a shows the wavelet transform dynamic spectrum for the radial electric field. The intermittent series of power maxima are clearly visible around the predicted GAM frequency of $\sim 10 \mathrm{kHz}$. The integrated wavelet spectrum of electric field fluctuations is shown in Figure $3 \mathrm{~b}$. The spectral peak at $9.3 \mathrm{kHz}$ is approximately 4 times above the power level of turbulent fluctuations at neighboring frequencies. We noted an apparent additional spectral peak at $\sim 15 \mathrm{kHz}$ and a number of smaller maxima at frequencies below $\sim 5 \mathrm{kHz}$. We also showed the Fourier power spectrum estimation for the same signal in the red trace, which reveals multiple peaks within a single broad spectral peak of a wavelet spectrum estimate. These additional spectral peaks in the Fourier-based spectrum are separated from the main peak by no more than $1 \mathrm{kHz}$. The Fourier spectral density also shows a number of higher harmonics, at 15,19 and $23 \mathrm{kHz}$. The nonlinear interactions between the modes represented by the peaks clustering around $\sim 10 \mathrm{kHz}$, could lead to low-frequency modulation in the electric field signal.

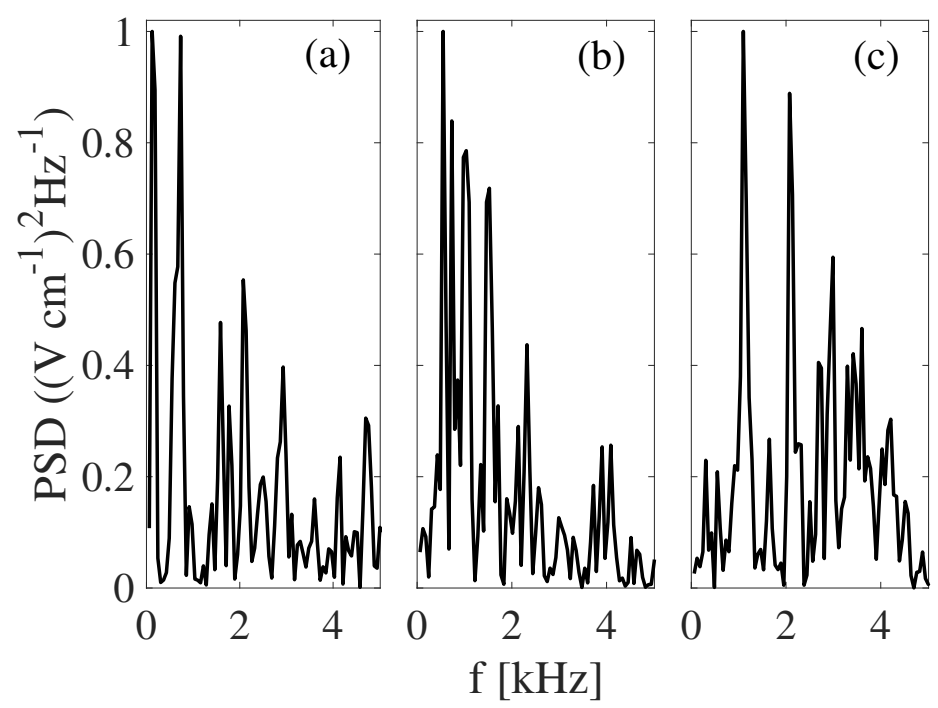

Figure 5. Fourier spectra of: (a) turbulence envelope $E_{T}$, (b) GAM envelope $E_{G}$, and (c) of LFZFs signal $S_{Z}$.

In order to study the GAM amplitude modulation and its possible impact on turbulence and ZFs, we decomposed our data into three components with distinct temporal scales: turbulence, GAM, and LFZF. We used the EEMD technique to generate $25 \mathrm{IMFs}$ from the original, $E_{r}$ data, with the largest frequency of about $\sim 115 \mathrm{kHz}$ and the smallest frequency at $\sim 70 \mathrm{~Hz}$. The largest frequency is treated as the residual noise in the data and is discarded. Similarly, we discarded the smallest frequency mode, which is a nonlinear trend very close to zero. We then combined the IMFs at three different frequency ranges to obtain signals of interest. The turbulence, $S_{T}(t)$, is a superposition of IMFs $2-4$, corresponding to mean instantaneous frequencies between $\sim 25 \mathrm{kHz}$ and $\sim 66 \mathrm{kHz}$. The GAM is a single IMF with the mean frequency of 10 $\mathrm{kHz}, S_{G}(t)$. Finally, the LFZFs signal $S_{Z}(t)$ is obtained by summing modes 8-20, with mean frequencies in the range 77-5000 Hz. We apply an analytic signal approach to $S_{T}$ and $S_{G}$, in order to obtain their 
amplitude modulation envelopes $E_{T}$ and $E_{G}$, respectively. The turbulence envelope is smoothed over 50 neighboring points. Three panels in Figure 4 shows signals $S_{T}, S_{G}, S_{Z}$ and the upper envelopes $E_{T}$ and $E_{G}$. The envelopes $E_{T}$ and $E_{G}$ were smoothed over 50 and 30 neighboring points, respectively, and shifted vertically by 10 units for clarity. A close visual inspection indicates that there is no phase coherence between turbulence envelope, GAM envelope and the LFZF signal. This is confirmed by a linear cross correlation coefficients calculated for the pairs $\left(E_{T}, E_{G}\right),\left(E_{T}, S_{Z}\right)$ and $\left(E_{G}, S_{Z}\right)$, which had values at around or below 0.25 , at different non-zero time lags.

While there is no phase coherence in the LF behavior of $E_{T}, E_{G}$, and $S_{Z}$, there is a considerable spectral coherence for these time traces. Figure $5 \mathrm{a}-\mathrm{c}$ shows the Fourier spectral estimate of power for $E_{T}, E_{G}$, and $S_{Z}$, respectively. Note that all spectra have been normalized to their respective maxima, which had a value of 0.05 for turbulence envelope, 0.28 for the GAM envelope, and 0.008 for the LFZF signal. All spectra have pronounced peaks at about $1 \mathrm{kHz}$ and at $2 \mathrm{kHz}$. The GAM envelope spectrum shows a number of peaks at relatively constant increments, positioned at $\sim 0.55, \sim 0.75,1$ and $1.5 \mathrm{kHz}$. We also note that, the LFZF spectrum shows a broad spectral power between frequencies $3-5 \mathrm{kHz}$. This is a strong suggestion that there are nonlinear interactions between various modes, close to the GAM frequency, and also non-local between GAM/turbulence and LFZF.

The resonant three wave interaction process is considered as a model of coupling between different modes present in the radial electric field time series. For a single point time series measurements, we can only consider frequency resonances $f_{3}=f_{1}+f_{2}$. The strength of these interactions is then approximated by a bi-coherence, which we have calculated using wavelet coefficients and averaged over all times. Figure 6 shows only positive frequency part of the bi-coherence, which was thresholded at a relatively high value of 0.7 to emphasize the most relevant interactions. We find the signature of strong self-interactions at the GAM frequencies $f_{1} \approx f_{2} \approx 10 \mathrm{kHz}$, positioned on the diagonal line, as well as interactions with the LF modes, such as $f_{1}=8.4 \mathrm{kHz}, f_{2}=1.2 \mathrm{kHz}$, non-local in frequency space. Interestingly, the bi-coherence also reveals the importance of a mode with $f \approx 4 \mathrm{kHz}$, which also self-interacts and couples to LF modes.

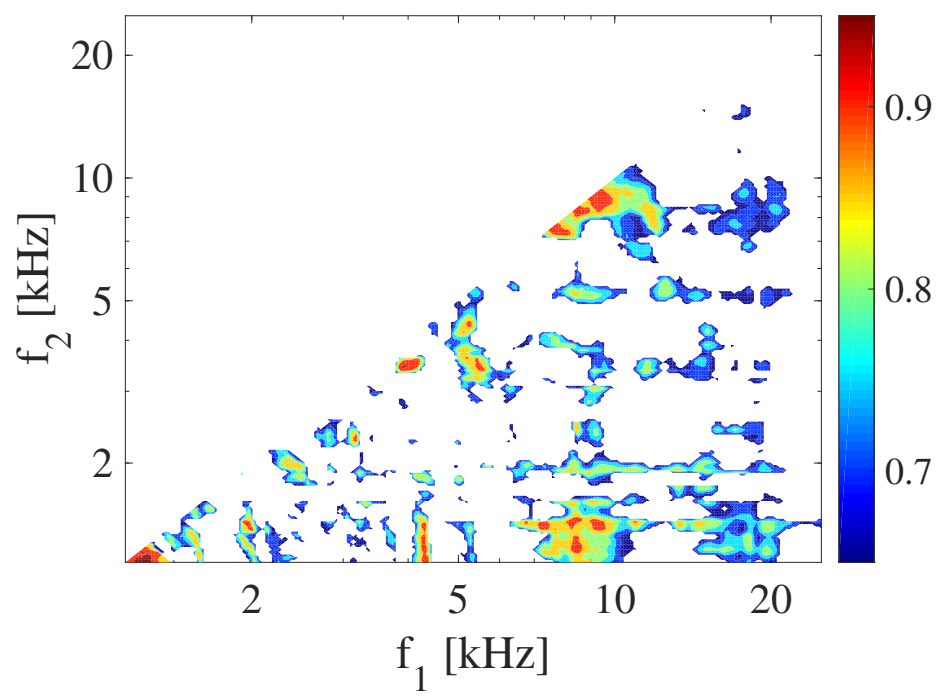

Figure 6. The wavelet bi-coherence for the entire data set $E_{r}$. 


\section{Discussion}

We performed the analysis of the fluctuations in the radial electric field component obtained from the reciprocating Langmuir Mach probe at the mid plane of MAST. Wavelet dynamic spectrum revealed temporal power intermittency of the GAM, while the Fourier spectrum estimated shows multiple spectral peaks in the vicinity of $10 \mathrm{kHz}$. We then tested the hypothesis that the GAM amplitude modulation was due to self-interaction of the GAM, and that the LF component further mediated the energy exchange with turbulent modes. We used an HT based technique, EEMD, to extract radial electric field fluctuations on temporal scales of turbulence, the GAM and LFZF. Envelopes of turbulent signal and the GAM were constructed using analytic signal approach. We found a significant spectral coherence for the turbulent envelope, GAM envelope, and the LF component. The bi-coherence revealed strong nonlinear interactions, local self-interactions near the GAM frequency [34], and non-local interactions with LF mediated by the GAM. This is broadly consistent with previous results presented in the literature $[28,35]$.

Our findings may be of particular importance for better understanding how the presence of the GAM alters the physics of L-H transition. It has been reported that the LF component, which we have termed LFZF in this work, increases significantly at the expense of the GAM during the transition [24]. The energy flow for the three component system, turbulence, GAM, and ZF, is often modeled using a nonlinear predator-prey type formulation $[2,36]$. These models incorporate all key physical interactions important to the dynamics of the system but retain simplicity that allows better understanding of how each component affects their collective complex dynamics, for example, the L-H transition. The behavior of these models is strongly influenced by the included interactions between various components. This work clearly shows that in addition to the linear impact of the GAM on ZFs [37], the nonlinear interactions are also important.

Author Contributions: Conceptualization, B.H.; methodology, B.H.; data curation, N.W. and MAST Team.

Funding: B.H. acknowledges funding from EUROfusion agreement number 2007792. N.W. gratefully acknowledges funding and support through EUROfusion researcher grant AWP16-ERG-CCFE/Walkden.

Acknowledgments: This work has been carried out within the framework of the EUROfusion Consortium and has received funding from the Euratom research and training programme 2014-2018 under grant agreement No. 633053 and from the RCUK Energy Programme [grant number EP/P012450/1]. The views and opinions expressed herein do not necessarily reflect those of the European Commission.

Conflicts of Interest: The authors declare no conflict of interest.

\section{References}

1. Zweben, S.J.; Boedo, J.A.; Grulke, O.; Hidalgo, C.; LaBombard, B.; Maqueda, R.J.; Scarin, P.; Terry, J.L. Edge turbulence measurements in toroidal fusion devices. Plasma Phys. Control. Fus. 2007, 49, S1. [CrossRef]

2. Diamond, P.H.; Itoh, S.I.; Itoh, K.; Hahm, T.S. Zonal flows in plasma-A review. Plasma Phys. Control. Fus. 2005, 47, R35-R161. [CrossRef]

3. Rosenbluth, M.N.; Hinton, F.L. Poloidal flow driven by ion-temperature-gradient turbulence in tokamaks. Phys. Rev. Lett. 1998, 80, 724-727. [CrossRef]

4. Dimits, A.M.; Bateman, G.; Beer, M.A.; Cohen, B.I.; Dorland, W.; Hammett, G.W.; Kim, C.; Kinsey, J.E.; Kotschenreuther, M.; Kritz, A.H.; et al. Comparisons and physics basis of tokamak transport models and turbulence simulations. Phys. Plasmas 2000, 7, 969-983. [CrossRef]

5. Numata, R.; Ball, R.; Dewar, R.L. Bifurcation in electrostatic resistive drift wave turbulence. Phys. Plasmas 2007, 26, 102312. [CrossRef]

6. Gadgil, S.; Hnat, B.; Rowlands, G. Investigation of drift-wave instability in the presence of zonal flows using spatial averaging. Phys. Plasmas 2019, 26, 012105. [CrossRef]

7. Lin, Z.; Hahm, T.S.; Lee, W.W.; Tang, W.M.; White, R.B. Turbulent transport reduction by zonal flows: Massively parallel simulations. Science 1998, 281, 1835-1837. [CrossRef] 
8. Moyer, R.A.; Tynan, G.R.; Holland, C.; Burin, M.J. Increased Nonlinear Coupling between Turbulence and Low-Frequency Fluctuations at the L-H Transition. Phys. Rev. Lett. 2001, 87, 135001. [CrossRef] [PubMed]

9. Tynan, G.R.; Moyer, R.A.; Burin, M.J.; Holland, C. On the nonlinear turbulent dynamics of shear-flow decorrelation and zonal flow generation. Phys. Plasmas 2001, 8, 2691-2699. [CrossRef]

10. Winsor, N.; Johnson, J.L.; Dawson, J.M. Geodesic acoustic waves in hydromagnetic systems. Phys. Fluids 1968, 11, 2448-2450. [CrossRef]

11. Gao, Z. Collisional damping of the geodesic acoustic mode. Phys. Plasmas 2013, 20, 032501. [CrossRef]

12. Jakubowski, M.; Fonck, R.J.; McKee, G.R. Observation of coherent sheared turbulence flows in the DIII-D tokamak. Phys. Rev. Lett. 2002, 89, 265003. [CrossRef] [PubMed]

13. Silva, C.; Arnoux, G.; Groth, M.; Hidalgo, C.; Marsen, S.; JET-EFDA Contributors. Observation of geodesic acoustic modes in the JET edge plasma. Plasma Phys. Control. Fus. 2012, 55, 025001. [CrossRef]

14. Ido, T.; Miura, Y.; Kamiya, K.; Hamada, Y.; Hoshino, K.; Fujisawa, A.; Itoh, K.; Itoh, S.I.; Nishizawa, A.; Ogawa, H.; et al. Geodesic acoustic mode in JFT 2M tokamak plasmas. Plasma Phys. Control. Fus. 2006, 48, S41-S50. [CrossRef]

15. Robinson, J.R.; Hnat, B.; Dura, P.; Kirk, A.; Tamain, P.; MAST Team. Interaction between a low-frequency electrostatic mode and resonant magnetic perturbations in MAST. Plasma Phys. Control. Fus. 2012, 54, 105007. [CrossRef]

16. Robinson, J.R.; Hnat, B.; Thyagaraja, A.; McClements, K.G.; Knight, P.J.; Kirk, A.; MAST Team. Global two-fluid simulations of geodesic acoustic modes in strongly shaped tight aspect ratio tokamak plasmas. Phys. Plasmas 2013, 20, 052302. [CrossRef]

17. Itoh, K.; Itoh, S.I.; Diamond, P.H.; Fujisawa, A.; Yagi, M.; Watari, T.; Nagashima, Y.; Fukuyama, A. Geodesic acoustic eigenmodes. Plasma Fus. Res. 2006, 1, 037. [CrossRef]

18. Miyato, N.; Kishimoto, Y.; Li, J.Q. Nonlocal behaviour of zonal flows in tokamak plasmas. Plasma Phys. Control. Fus. 2006, 48, A335. [CrossRef]

19. Liu, A.D.; Lan, T.; Yu, C.X.; Zhao, H.L.; Yan, L.W.; Hong, W.Y.; Dong, J.Q.; Zhao, K.J.; Qian, J.; Cheng, J.; et al. Characterizations of low-frequency zonal flow in the edge plasma of the HL-2A tokamak. Phys. Rev. Lett. 2009, 103, 095002. [CrossRef]

20. Hnat, B.; Gadgil, S.; Kirk, A.; Militello, F.; Walkden, N.; MAST Team. Experimental constraint on the radial mode number of the geodesic acoustic mode from multi-point Langmuir probe measurements in MAST Ohmic plasma. Plasma Phys. Controll. Fus. 2018, 60, 085016. [CrossRef]

21. Rogers, B.N.; Dorland, W.; Kotschenreuther, M. Generation and stability of zonal flows in ion-temperaturegradient mode turbulence. Phys. Rev. Lett. 2000, 85, 5336-5339. [CrossRef] [PubMed]

22. Scott, B. The geodesic transfer effect on zonal flows in tokamak edge turbulence. Phys. Lett. A 2003, 320, 53-62. [CrossRef]

23. Fujisawa, A.; Shimizu, A.; Nakano, H.; Ohshima, S.; Itoh, K.; Nagashima, Y.; Itoh, S.I.; Iguchi, H.; Yoshimura, Y.; Minami, T.; et al. Intermittent characteristics in coupling between turbulence and zonal flows. Plasma Phys. Control. Fus. 2007, 49, 211-217. [CrossRef]

24. Team, A.U.; Conway, G.D.; Angioni, C.; Ryter, F.; Sauter, P.; Vicente, J. Mean and oscillating plasma flows and turbulence interactions across the L-H confinement transition. Phys. Rev. Lett. 2011, 106, 065001.

25. MacLatchy, C.S.; Boucher, C.; Poirier, D.A.; Gunn, J. Gundestrup: A Langmuir/Mach probe array for measuring flows in the scrape-off layer of TdeV. Rev. Sci. Instrum. 1992, 63, 3923-3929. [CrossRef]

26. Tamain, P.; Kirk, A.; Nardon, E.; Dudson, B.; Hnat, B. Edge turbulence and flows in the presence of resonant magnetic perturbations on MAST. Plasma Phys. Control. Fus. 2010, 52, 075017. [CrossRef]

27. Scannell, R.; Walsh, M.J.; Carolan, P.G.; Darke, A.C.; Dunstan, M.R.; Huxford, R.B.; McArdle, G.; Morgan, D.; Naylor, G.; O'Gorman, T.; et al. Design of a new Nd: YAG Thomson scattering system for MAST. Rev. Sci. Instrum. 2008, 79, 10E730. [CrossRef] [PubMed]

28. Liu, Y.W. Hilbert transform and applications. In Fourier Transform Applications; Salih, S., Ed.; Intech: Barcelona, Spain, 2012; ISBN 978-953-51-0518-3. 
29. Huang, N.E.; Shen, Z.; Long, S.R.; Wu, M.C.; Shih, H.H.; Zheng, Q.; Yen, N.C.; Tung, C.C.; Liu, H.H. The empirical mode decomposition and the Hilbert spectrum for nonlinear and non-stationary time series analysis. Proc. R. Soc. Lond. Ser. A. Math. Phys. Eng. Sci. 1998, 454A, 903-995. [CrossRef]

30. Wu, Z.; Huang, N.E. Ensemble empirical mode decomposition: A noise-assisted data analysis method. Adv. Adapt. Data Anal. 2009, 1, 1-41. [CrossRef]

31. Torres, M.E.; Colominas, M.A.; Schlotthauer, G.; Flandrin, P. A complete ensemble empirical mode decomposition with adaptive noise. In Proceedings of the 36th IEEE International Conference on Acoustics, Speech and Signal Process, ICASSP, Prague, Czech Republic, 22-27 May 2011; pp. 4144-4147.

32. Matlab Documentation-MathWorks. Available online: https://uk.mathworks.com/help/wavelet/ref/cwtftinfo. html (accessed on 7 May 2019).

33. Torrence, C.; Compo, G.P. A practical guide to wavelet analysis. Bull. Am. Soc. 1998, 79, 61-78. [CrossRef]

34. Sasaki, M.; Itoh, K.; Nagashima, Y.; Ejiri, A.; Takase, Y. Nonlinear self-interaction of geodesic acoustic modes in toroidal plasmas. Phys. Plasmas 2009, 16, 022306. [CrossRef]

35. Ramisch, M.; Stroth, U.; Niedner, S.; Scott, B. On the detection of Reynolds stress as a driving and damping mechanism of geodesic acoustic modes and zonal flows. New J. Phys. 2003, 4, 1-12. [CrossRef]

36. Miki, K.; Diamond, P.H. Role of the geodesic acoustic mode shearing feedback loop in transport bifurcations and turbulence spreading. Phys. Plasmas 2010, 17, 032309. [CrossRef]

37. Hallatschek, K.; Biskamp, D. Transport control by coherent zonal flows in the core/edge transitional regime. Phys. Rev. Lett. 2001, 12, 1223-1227. [CrossRef]

(C) 2019 by the authors. Licensee MDPI, Basel, Switzerland. This article is an open access article distributed under the terms and conditions of the Creative Commons Attribution (CC BY) license (http:/ / creativecommons.org/licenses/by/4.0/). 